\title{
Dream stream decoded
}

Machine learning and imaging show what the dreaming brain sees.

Dreaming shows how busy the brain stays during sleep. A research team in Japan has now found a way to infer dreams from brain activity. "Dreaming is a fascinating phenomenon because it often accompanies visual experience, but there is no physical sensory input," says Yukiyasu Kamitani from ATR Computational Neuroscience Laboratories, who led the work. "When a person is dreaming about an object or scene, the brain activity is similar to that caused by actually seeing the same object or scene."

The scientists recruited people to their sleep lab and used functional magnetic resonance (fMRI) imaging to decode dreams indirectly. Sleeping test subjects were repeatedly wakened when vital signs revealed they were dreaming. The test subjects recounted their dreams to the investi- gators, who transcribed the accounts and then matched terms to a lexical database.

Using these terms, the team mined image databases to compile visuals that approximated the dreams. They measured brain activity when awake study participants looked at these image compilations.

fMRI reveals neural circuit activity through changes in blood flow and oxygenation, but the naked eye cannot discern differences in activity between dreaming brains and brains processing visual summaries of dreams. "That's why we need pattern recognition by machine learning," says Kamitani. The scientists created a computational approach to compare and contrast the changes in grayscale pixel intensity in the fMRI images.

The team developed mathematical formulas called decoders to read out dream content from fMRI data. Detailed activity patterns are specific to individuals, so the decoders had to be personalized. "A decoder built for one person does not generalize to another person," says Kamitani.

To recognize patterns in the data, the researchers applied a machine learning algorithm, which they trained on the fMRI data from awake subjects looking at the image compilations. This approach linked neural activity and dream content.

Kamitani now plans to expand his dream research to look at basic visual features such as color and shape. "It may also be possible to decode contents related to action and emotion." In these experiments, participants must fall asleep in an MRI scanner, which is not so hard, says Kamitani. Stage fright gives way to boredom, and it becomes difficult to stay awake.

\section{Vivien Marx}

RESEARCH PAPERS

Horikawa, T. et al. Neural decoding of visual imagery during sleep. Science 340, 639-642 (2013). 